\title{
STABILITY AND THE INDEX OF BIHARMONIC HYPERSURFACES IN A RIEMANNIAN MANIFOLD
}

\author{
YE-LIN OU *
}

\begin{abstract}
In this paper, we give an explicit second variation formula for a biharmonic hypersurface in a Riamannian manifold similar to that of a minimal hypersurface. We then use the second variation formula to compute the stability index of the known biharmonic hypersurfaces in a Euclidean sphere, and to prove the non-existence of unstable proper biharmonic hypersurface in a Euclidean space or a hyperbolic space, which adds another special case to support Chen's conjecture on biharmonic submanifolds.
\end{abstract}

\section{Stability AND THE INDEX OF MiNIMAL HYPERSURFACES}

It is well known that minimal hypersurfaces $M^{m} \rightarrow\left(N^{m+1}, h\right)$ in a Riemannian manifold are critical points of the area functional on hypersurfaces, i.e.,

$$
\frac{\mathrm{d}}{\mathrm{d} t}\left(\operatorname{Area}\left(M_{t}\right)\right)_{t=0}=-m \int_{M} f H \mathrm{~d} v_{g}=0 .
$$

This is equivalent to the statement that the mean curvature $H=\frac{1}{m} \operatorname{Tr} A$ of the hypersurface of $M$ vanishes identically, where $A$ is the shape operator of the hypersurface.

As it is also well known that a critical point may not give a local minimum of the area functional. To have a better understanding of minimal hypersurfaces as the critical points of a functional, one needs to know the second variation that leads to the concepts of the stability and the index of minimal hypersurfaces.

Date: $02 / 08 / 2020$.

1991 Mathematics Subject Classification. 58E20.

Key words and phrases. The second variations of biharmonic hypersurfaces, Stable biharmonic hypersurfaces, The index of biharmonic hypersurfaces, the index of biharmonic torus, constant mean curvature hypersurfaces.

* This work was supported by a grant from the Simons Foundation (\#427231, Ye-Lin Ou). 
Recall (see e.g., [1] and [10]) that a minimal hypersurface is stable if the second variation of the area functional is always nonnegative for any normal variation with compact support, i.e.,

$$
\frac{\mathrm{d}^{2}}{\mathrm{~d} t^{2}}\left(\operatorname{Area}\left(M_{t}\right)\right)_{t=0} \geq 0
$$

For a complete orientable minimal hypersurface $M^{m} \rightarrow\left(N^{m+1}, h\right)$ in a Riemannian manifold, there is a unit normal vector field $\xi$ along $M$ so that any section $V$ of the normal bundle with compact support can be written as $V=f \xi$ for a function $f$ with compact support in $M$, and the second variation of the area functional with the $V=f \xi$ as variation vector field can be written as :

$$
\frac{\mathrm{d}^{2}}{\mathrm{~d} t^{2}}\left(\operatorname{Area}\left(M_{t}\right)\right)_{t=0}=\int_{M}\left\{|\nabla f|^{2}-\left(\operatorname{Ric}^{N}(\xi, \xi)+|A|^{2}\right) f^{2}\right\} \mathrm{d} v_{g}
$$

where $|A|^{2}$ is the squared norm of the second fundamental form of the hypersurafce, and $\operatorname{Ric}^{N}(\xi, \xi)=\sum_{i=1}^{m}\left\langle\mathrm{R}^{N}\left(\xi, e_{i}\right) e_{i}, \xi\right\rangle=\sum_{i=1}^{m} \mathrm{R}^{N}\left(\xi, e_{i}, \xi, e_{i}\right)$ is the Ricci curvature in the direction $\xi$.

Note that by using the divergence theorem: $\int_{M} f \Delta f \mathrm{~d} v_{g}=-\int_{M}|\nabla f|^{2} \mathrm{~d} v_{g}$, we can rewrite (10) as

$$
\frac{\mathrm{d}^{2}}{\mathrm{~d} t^{2}}\left(\operatorname{Area}\left(M_{t}\right)\right)_{t=0}=q_{M}(f)=-\int_{M} f J(f) \mathrm{d} v_{g} \geq 0,
$$

where $J(f)=\Delta f+\left(|A|^{2}+\operatorname{Ric}^{N}(\xi, \xi)\right) f$ is called the Jacobi operator on the minimal hypersurface.

Recall (see e.g., [1]) that the index of a minimal hypersurface $M$, denoted by $\operatorname{Ind}(M)$, is the maximum dimension of any subspace $V$ of $C_{0}^{\infty}(M)$ on which $q_{M}(f)$ is negative, i.e.,

$$
\operatorname{Ind}(M)=\operatorname{Max}\left\{\operatorname{dim} V: V \subset C_{0}^{\infty}(M) \mid q_{M}(f)<0, \forall f \in V\right\} .
$$

In particular, the index of a minimal hypersurface $M \hookrightarrow S^{m+1}$ in a Euclidean sphere is the the largest dimension of subspace $V \subset C_{0}^{\infty}(M)$ on which the quadratic form

$$
q_{M}(f)=-\int_{M} f\left[\Delta f+\left(|A|^{2}+m\right) f\right] \mathrm{d} v_{g}<0 .
$$

The following are some well known facts about the index of minimal hypersurfaces in a sphere:

- For a compact minimal hypersurface $M$ in $S^{m+1}$, $\operatorname{Ind}(M) \geq 1$ and with " = " holds if and only if $M$ is a totally geodesic equator $S^{m} \subset S^{m+1}([14])$; 
- For a compact non-totally geodesic minimal hypersurface $M^{m} \rightarrow S^{m+1}$, $\operatorname{Ind}(M) \geq m+3$ (see [16] for $m=2$ and [15] for the general case);

- For the minimal Clifford torus $S^{p}\left(\sqrt{\frac{p}{m}}\right) \times S^{q}\left(\sqrt{\frac{q}{m}}\right) \hookrightarrow S^{m+1}$ with $p+q=$ $m$, the index $\operatorname{Ind}(M)=m+3$;

- It has been a conjecture which is still open (see e.g., [1, [2]) that any compact non-totally geodesic minimal hypersurface $M^{m} \rightarrow S^{m+1}$ with $\operatorname{Ind}(M)=m+3$ is a Clifford torus.

Biharmonic hypersurfaces are generalizations of minimal hypersurfaces. A biharmonic hypersurface in a Riemannian manifold can be characterized as an isometric immersion $M^{m} \rightarrow\left(N^{m+1}, h\right)$ whose mean curvature function $H$ solves the following equation (see [8], 6] and [5] for the case when the ambient space is a space form, and [12] for the general case):

$$
\left\{\begin{array}{l}
\Delta H-H|A|^{2}+H \operatorname{Ric}^{N}(\xi, \xi)=0, \\
2 A(\operatorname{grad} H)+\frac{m}{2} \operatorname{grad} H^{2}-2 H\left(\operatorname{Ric}^{N}(\xi)\right)^{\top}=0,
\end{array}\right.
$$

where $\operatorname{Ric}^{N}: T_{q} N \longrightarrow T_{q} N$ denotes the Ricci operator of the ambient space defined by $\left\langle\operatorname{Ric}^{N}(Z), W\right\rangle=\operatorname{Ric}^{N}(Z, W)$ and $A$ is the shape operator of the hypersurface with respect to the unit normal vector $\xi$.

It is clear from (44) that any minimal hypersurface is automatically a biharmonic hypersurface. So it is a custom to call a biharmonic hypersurface which is not minimal a proper biharmonic hypersurface. For more study of biharmonic maps and biharmonic submanifolds we refer the reader to a recent book [13] and the references therein.

In this paper, we derive an explicit second variation formula for a biharmonic hypersurface in a Riemannian manifold similar to that of a minimal hypersurface. We then use the second variation formula to compute the stability index of the known biharmonic hypersurfaces in a Euclidean sphere, and to prove the nonexistence of unstable proper biharmonic hypersurface in a Euclidean space or a hyperbolic space, which adds another special case to support Chan's conjecture on biharmonic submanifolds.

\section{Stability AND THE INDEX OF BIHARMONIC HyPERSURFACES}

In light of the ideas from the study of stability and the index of minimal hypersurfaces, we define a proper biharmonic hypersurface $M \rightarrow\left(N^{m+1}, h\right)$ to be stable if the second variation of the bienergy functional is always nonnegative for any normal variation with compact support. With this, we have 
Theorem 2.1. A complete orientable biharmonic hypersurface $\phi: M^{m} \rightarrow\left(N^{m+1}, h\right)$ of a Riemannian manifold is stable if and only if for any compactly supported function $f$ on $M$, we have

$$
\begin{aligned}
Q(f)= & \left.\frac{\mathrm{d}^{2}}{\mathrm{~d} t^{2}} E_{2}\left(\phi_{t}\right)\right|_{t=0} \\
= & \left.\int_{M}\left[f\left(|A|^{2}-\operatorname{Ric}^{N}(\xi, \xi)\right)-\Delta f\right]^{2}\right] \mathrm{d} v_{g} \\
& +\int_{M}\left|m f \nabla H-2\left(\operatorname{Ric}^{N}(\xi)\right)^{\top}+2 A(\nabla f)\right|^{2} \mathrm{~d} v_{g} \\
& \left.+\int_{M} m f^{2} H\left[\left(\nabla_{\xi}^{N} \operatorname{Ric}^{N}\right)(\xi, \xi)\right)-2 \operatorname{Tr} \mathrm{R}^{N}\left(\xi, \cdot, \xi, \nabla_{\xi}^{N}(\cdot)\right)\right] \mathrm{d} v_{g} \\
& -\int_{M} 4 m f^{2} H \operatorname{Tr} \mathrm{R}^{N}(\xi, A(\cdot), \xi, \cdot) \mathrm{d} v_{g} \geq 0 .
\end{aligned}
$$

Proof. The following second variation formula for a general biharmonic map $\phi$ : $\left(M^{m}, g\right) \rightarrow\left(N^{n}, h\right)$ between two Riemannian manifolds was derived by Jiang in [7:

$$
\begin{aligned}
\left.\frac{\mathrm{d}^{2}}{\mathrm{~d} t^{2}} E_{2}\left(\phi_{t}\right)\right|_{t=0} & =\int_{M}\left[\left|J^{\phi}(V)\right|^{2}+\mathrm{R}^{N}(V, \tau(\phi), V, \tau(\phi)] \mathrm{d} v_{g}\right. \\
& -\sum_{i=1}^{m} \int_{M}\left\langle V,\left(\nabla_{\mathrm{d} \phi\left(e_{i}\right)}^{N} \mathrm{R}^{N}\right)\left(\mathrm{d} \phi\left(e_{i}\right), \tau(\phi)\right) V\right. \\
& +\left(\nabla_{\tau(\phi)}^{N} \mathrm{R}^{N}\right)\left(\mathrm{d} \phi\left(e_{i}\right), V\right) \mathrm{d} \phi\left(e_{i}\right) \\
& \left.+2 \mathrm{R}^{N}\left(\mathrm{~d} \phi\left(e_{i}\right), V\right) \nabla_{e_{i}}^{\phi} \tau(\phi)+2 \mathrm{R}^{N}\left(\mathrm{~d} \phi\left(e_{i}\right), \tau(\phi)\right) \nabla_{e_{i}}^{\phi} V\right\rangle \mathrm{d} v_{g} .
\end{aligned}
$$

When $\phi: M^{m} \rightarrow\left(N^{m+1}, h\right)$ is an orientable biharmonic hypersurface, we consider the normal variation with variation vector field $V=f \xi$ and use $\tau(\phi)=$ $m H \xi$, identify $\mathrm{d} \phi\left(e_{i}\right)=e_{i}$, and a straightforward computation to have

(8) $\sum_{i=1}^{m}\left\langle V,\left(\nabla_{\tau(\phi)}^{N} \mathrm{R}^{N}\right)\left(\mathrm{d} \phi\left(e_{i}\right), V\right) \mathrm{d} \phi\left(e_{i}\right)\right\rangle=m f H \sum_{i=1}^{m}\left\langle\xi,\left(\nabla_{\xi}^{N} \mathrm{R}^{N}\right)\left(e_{i}, f \xi\right) e_{i}\right\rangle$

$$
\left.=m f^{2} H\left[-\left(\nabla_{\xi}^{N} \operatorname{Ric}^{N}\right)(\xi, \xi)\right)+2 \operatorname{Tr} \mathrm{R}^{N}\left(\xi, \cdot, \xi, \nabla_{\xi}^{N}(\cdot)\right)\right],
$$

(9) $\sum_{i=1}^{m}\left\langle V,\left(\nabla_{\mathrm{d} \phi\left(e_{i}\right)}^{N} \mathrm{R}^{N}\right)\left(\mathrm{d} \phi\left(e_{i}\right), \tau(\phi)\right) V\right\rangle=m f^{2} H \sum_{i=1}^{m}\left\langle\xi,\left(\nabla_{e_{i}}^{N} \mathrm{R}^{N}\right)\left(e_{i}, \xi\right) \xi\right\rangle=0$, 
STABILITY AND THE INDEX OF BIHARMONIC HYPERSURFACES IN A RIEMANNIAN MANIFOLIפ

$$
\begin{aligned}
& \sum_{i=1}^{m}\left\langle V, 2 \mathrm{R}^{N}\left(\mathrm{~d} \phi\left(e_{i}\right), V\right) \nabla_{e_{i}}^{\phi} \tau(\phi)\right\rangle=2 m f^{2} H \sum_{i=1}^{m} \mathrm{R}^{N}\left(\xi, \nabla_{e_{i}}^{N} \xi, e_{i}, \xi\right) \\
& =2 m f^{2} H \operatorname{Tr} \mathrm{R}^{N}(\xi, A(\cdot), \xi, \cdot), \text { and } \\
& \sum_{i=1}^{m}\left\langle V, 2 \mathrm{R}^{N}\left(\mathrm{~d} \phi\left(e_{i}\right), \tau(\phi)\right) \nabla_{e_{i}}^{\phi} V\right\rangle=2 m f H \sum_{i=1}^{m}\left\langle\xi, \mathrm{R}^{N}\left(e_{i}, \xi\right) \nabla_{e_{i}}^{N}(f \xi)\right\rangle \\
& =2 m f^{2} H \operatorname{Tr} \mathrm{R}^{N}(\xi, A(\cdot), \xi, \cdot) .
\end{aligned}
$$

On the other hand, using the formula (see e.g., [11])

$$
J^{\phi}(V)=J^{\phi}(f \xi)=f J^{\phi}(\xi)-(\Delta f) \xi-2 \nabla_{\nabla f}^{\phi} \xi,
$$

and a further computation (see also [12]), we obtain

$$
\begin{gathered}
J^{\phi}(\xi)=-\sum_{i=1}^{m}\left(\left(\nabla_{e_{i}}^{\phi} \nabla_{e_{i}}^{\phi}-\nabla_{\nabla_{e_{i}}^{M} e_{i}}^{\phi}\right) \xi-\mathrm{R}^{N}\left(\mathrm{~d} \phi\left(e_{i}\right), \xi\right) \mathrm{d} \phi\left(e_{i}\right)\right) \\
=\left(|A|^{2}-\operatorname{Ric}^{N}(\xi, \xi)\right) \xi+m \nabla H-2\left(\operatorname{Ric}^{N}(\xi)\right)^{\top}, \\
2 \nabla_{\nabla f}^{\phi} \xi=-2 A(\nabla f) .
\end{gathered}
$$

It follows from (13), (14) and (12) that

$$
\left|J^{\phi}(V)\right|^{2}=\left|J^{\phi}(f \xi)\right|^{2}
$$

$$
=\left[f\left(|A|^{2}-\operatorname{Ric}^{N}(\xi, \xi)\right)-\Delta f\right]^{2}+\left|m f \nabla H-2\left(\operatorname{Ric}^{N}(\xi)\right)^{\top}+2 A(\nabla f)\right|^{2} .
$$

Substituting (7)-(11) and (15) into (6) we obtain

$$
\begin{aligned}
Q(f)= & \left.\frac{\mathrm{d}^{2}}{\mathrm{~d} t^{2}} E_{2}\left(\phi_{t}\right)\right|_{t=0} \\
= & \left.\int_{M}\left[f\left(|A|^{2}-\operatorname{Ric}^{N}(\xi, \xi)\right)-\Delta f\right]^{2}\right] \mathrm{d} v_{g} \\
& +\int_{M}\left|m f \nabla H-2\left(\operatorname{Ric}^{N}(\xi)\right)^{\top}+2 A(\nabla f)\right|^{2} \mathrm{~d} v_{g} \\
& \left.+\int_{M} m f^{2} H\left[\left(\nabla_{\xi}^{N} \operatorname{Ric}^{N}\right)(\xi, \xi)\right)-2 \operatorname{Tr} \mathrm{R}^{N}\left(\xi, \cdot, \xi, \nabla_{\xi}^{N}(\cdot)\right)\right] \mathrm{d} v_{g} \\
& -\int_{M} 4 m f^{2} H \operatorname{Tr} R^{N}(\xi, A(\cdot), \xi, \cdot) \mathrm{d} v_{g},
\end{aligned}
$$

from which and the definition of the stability of a biharmonic hypersurface we obtain the theorem.

Corollary 2.2. An orientable biharmonic hypersurface $\phi: M^{m} \rightarrow\left(N^{m+1}(c), h\right)$ in a space form of constant sectional curvature $c$ is stable if and only if the stability 
inequality $Q(f) \geq 0$ holds for any compactly supported smooth function $f$ on $M$, where

$$
Q(f)=\int_{M}\left\{\left[f\left(|A|^{2}-c m\right)-\Delta f\right]^{2}+|m f \nabla H+2 A(\nabla f)|^{2}-4 c m^{2} f^{2} H^{2}\right\} \mathrm{d} v_{g} .
$$

In particular, (i) any biharmonic hypersurface in a Euclidean space or a hyperbolic space is stable, and (ii) the stability quadratic form for a biharmonic hypersurface $M^{m} \rightarrow S^{m+1}$ in a Euclidean sphere is given by

$$
Q(f)=\int_{M}\left\{\left[f\left(|A|^{2}-m\right)-\Delta f\right]^{2}+|m f \nabla H+2 A(\nabla f)|^{2}-4 m^{2} f^{2} H^{2}\right\} \mathrm{d} v_{g} .
$$

Proof. The corollary follows from Equation (16) and the following identities for a space form $N^{m+1}(c)$ of constant sectional curvature $c$ :

$$
\begin{aligned}
& \left.\operatorname{Ric}^{N}(\xi, \xi)\right)=m c, \\
& \left.\operatorname{Ric}^{N}(\xi)\right)^{\top}=0, \\
& \left(\nabla_{\xi}^{N} \operatorname{Ric}^{N}\right)(\xi, \xi)=0, \\
& \operatorname{Tr} \operatorname{R}^{N}\left(\xi, \cdot, \xi, \nabla_{\xi}^{N}(\cdot)\right)=0, \text { and } \\
& 4 m f^{2} H \operatorname{Tr} \mathrm{R}^{N}(\xi, A(\cdot), \xi, \cdot)=4 c m^{2} f^{2} H^{2} .
\end{aligned}
$$

Remark 1. For stable minimal surfaces in Euclidean space $\mathbb{R}^{3}$, we have a wellknown result of do-Carmo-Peng: Any complete oriented and stable minimal surface $\phi: M^{2} \rightarrow \mathbb{R}^{3}$ is a plane. On the other hand, we know that catenoid is a complete and oriented minimal surfaces in $\mathbb{R}^{3}$, so it is unstable. In contrast, our corollary above says that there is no unstable biharmonic hypersurface in a Euclidean space or a hyperbolic space. This adds another special case to support Chen's conjecture on biharmonic submanifolds which can be stated as there exists no proper biharmonic submanifold in a Euclidean space. See [13] for a more detailed account on Chen's conjecture on biharmonic submanifolds.

\section{The STABILITY INDEX OF BIHARMONIC HYPERSURFACES IN $S^{m+1}$}

Again, following the idea of the index of minimal hypersurfaces, we define the index of a proper biharmonic hypersurface $M \rightarrow\left(N^{m+1}, h\right)$ to be the maximum dimension of any subspace $V$ of $C_{0}^{\infty}(M)$ on which $Q(f)$ defined in (16) is negative, i.e.,

$$
\operatorname{Ind}(M)=\operatorname{Max}\left\{\operatorname{dim} V: V \subset C_{0}^{\infty}(M) \mid Q(f)<0, \forall f \in V\right\} .
$$

About biharmonic hypersurfaces in a sphere, we know that 
- a hypersurface $\varphi:\left(M^{m}, g\right) \longrightarrow S^{m+1}$ with nonzero constant mean curvature is biharmonic if and only if the squared norm of the shape operator is constant (see [7] or directly using (44)).

- the only known proper biharmonic hypersurfaces in a sphere are ([7], [4]): $S^{m}\left(\frac{1}{\sqrt{2}}\right)$ and $S^{p}\left(\frac{1}{\sqrt{2}}\right) \times S^{m-p}\left(\frac{1}{\sqrt{2}}\right)$ for $p \neq \frac{m}{2}$, or an open part of one of these two. It has been a conjecture ([3]) which is still open that there is no other proper biharmonic hypersurface in a sphere than open parts of these two.

In this section, we will use the stability form (17) to compute the index of the known proper biharmonic hypersurfaces in a sphere.

Theorem 3.1. (i) For $1 \leq p<q=m-p$, the stability index of the proper biharmonbic hypersurface $S^{p}\left(\frac{1}{\sqrt{2}}\right) \times S^{q}\left(\frac{1}{\sqrt{2}}\right) \rightarrow S^{m+1}$ is

$$
\text { Ind }\left(S^{p}\left(\frac{1}{\sqrt{2}}\right) \times S^{q}\left(\frac{1}{\sqrt{2}}\right)\right)= \begin{cases}1, & \text { for } 1 \leq p<q \leq 2 p \\ p+2, & \text { for } 2 p<q .\end{cases}
$$

(ii) The stability index of the biharmonic hypersurface $S^{m}\left(\frac{1}{\sqrt{2}}\right) \rightarrow S^{m+1}$ is

$$
\text { Ind }\left(S^{m}\left(\frac{1}{\sqrt{2}}\right)\right)=1
$$

Proof. It is also known (cf. e.g., 1], 2]) that if $\lambda$ is an eigenvalue of the Laplacian on $S^{p}\left(r_{1}\right)$ with multiplicity $m_{\lambda}$ and $\mu$ is an eigenvalue of the Laplacian on $S^{m-p}\left(r_{2}\right)$ with multiplicity $m_{\mu}$, then $\nu=\lambda+\mu$ is an eigenvalue of the Laplacian on the product $S^{p}\left(r_{1}\right) \times S^{m-p}\left(r_{2}\right)$ with with a multiplicity $\sum m_{\lambda} m_{\mu}$ where the sum is made over all possible $\lambda, \mu$ satisfying $\lambda+\mu=\nu$.

In particular, when $r=1 / \sqrt{2}$, the proper biharmonic hypersurface $T^{p, q}:=$ $\left.S^{p}\left(\frac{1}{\sqrt{2}}\right) \times S^{m-p}\left(\frac{1}{\sqrt{2}}\right)\right) \rightarrow S^{m+1} \subset \mathbb{R}^{m+2}$ has two distinct principal curvatures $\lambda=$ -1 with multiplicity $m_{\lambda}=p$ and $\mu=1$ with multiplicity $m_{\mu}=m-p=q$. It follows that the mean curvature of the hypersurface $H=(m-2 p) / m$ is nonzero constant since $p \neq m / 2,|A|^{2}=m$, and $|A(\nabla f)|^{2}=|\nabla f|^{2}$. Substituting these into the stability index form (17) yields

$$
Q(f)=\int_{T^{p, q}}\left\{(\Delta f)^{2}-4 f \Delta f-4(q-p)^{2} f^{2}\right\} \mathrm{d} v_{g}
$$

For $1 \leq p<q=m-p \leq m-1$, the Laplacian on $S^{p}\left(\frac{1}{\sqrt{2}}\right) \times S^{q}\left(\frac{1}{\sqrt{2}}\right)$ has spectrum:

$$
\cdots<\cdots<\cdots<\lambda_{2}=-2 q<\lambda_{1}=-2 p<\lambda_{0}=0,
$$


For an eigenfunction $f$ with eigenvalue $\nu=-2 p$, i.e., $\Delta f=-2 p f$, (20) reads

$$
\begin{aligned}
Q(f) & =\int_{T^{p, q}}\left[4 p^{2}+8 p-4(q-p)^{2}\right] f^{2} \mathrm{~d} v_{g} \\
& =-4 \int_{T^{p, q}}\left(q^{2}-2 p q-2 p\right) f^{2} \mathrm{~d} v_{g} .
\end{aligned}
$$

It follows that $Q(f)<0$ if and only if the quadratic function $-4\left(q^{2}-2 p q-\right.$ $2 p)<0$, which is equivalent to $q>p+\sqrt{p^{2}+2 p}$. One can further check that $q>p+\sqrt{p^{2}+2 p}$ is equivalent to $q>2 p$ since there is no integer within $(2 p, p+$ $\left.\sqrt{p^{2}+2 p}\right)$.

On the other hand, for an eigenfunction $f$ of the second nonzero eigenvalue $\nu=-2 q$, i.e., $\Delta f=-2 q f$, (20) reads

$$
\begin{aligned}
Q(f) & =\int_{T^{p, q}}\left[4 q^{2}+8 q-4(q-p)^{2}\right] f^{2} \mathrm{~d} v_{g} \\
& >\int_{T^{p, q}} 4\left(2 q+p^{2}\right) f^{2} \mathrm{~d} v_{g}>0 .
\end{aligned}
$$

Similarly, we can check that $Q(f)$ is positive on any other eigenspace of the Laplacian on $S^{p}\left(\frac{1}{\sqrt{2}}\right) \times S^{q}\left(\frac{1}{\sqrt{2}}\right)$.

Finally, since $T^{p, q}=S^{p}\left(\frac{1}{\sqrt{2}}\right) \times S^{q}\left(\frac{1}{\sqrt{2}}\right)$ is compact, we have the following SturmLiouville's decomposition

$$
C^{\infty}\left(T^{p, q}\right)=\oplus_{i=0}^{\infty} E_{\lambda_{i}}
$$

where $E_{\lambda_{i}}$ denotes the eigenspace of the Laplacian on $S^{p}\left(\frac{1}{\sqrt{2}}\right) \times S^{q}\left(\frac{1}{\sqrt{2}}\right)$ with respect to the eigenvalue $\lambda_{i}$.

From this, together with the above discussion, we conclude that that for $1 \leq p<q=m-p \leq 2 p$, the largest subspace of smooth functions on the biharmonic hypersurface $S^{p}\left(\frac{1}{\sqrt{2}}\right) \times S^{q}\left(\frac{1}{\sqrt{2}}\right)$ on which $Q(f)<0$ is $E_{\lambda_{0}}$, the eigenspaces of the Laplacian on $S^{p}\left(\frac{1}{\sqrt{2}}\right) \times S^{q}\left(\frac{1}{\sqrt{2}}\right)$ corresponding the eigenvalues $\lambda_{0}=0$. Since $E_{\lambda_{0}}=\mathbb{R}$ has dimension 1. Thus, we obtain the first case in the index formula (18). For the case $1 \leq p<q=m-p$ and $q>2 p$, the largest subspace of smooth functions on the biharmonic hypersurface $S^{p}\left(\frac{1}{\sqrt{2}}\right) \times S^{q}\left(\frac{1}{\sqrt{2}}\right)$ on which $Q(f)<0$ is $E_{\lambda_{0}} \oplus E_{\lambda_{1}}$, where $E_{\lambda_{0}}$ and $E_{\lambda_{1}}$ are the eigenspaces of the Laplacian on $S^{p}\left(\frac{1}{\sqrt{2}}\right) \times S^{q}\left(\frac{1}{\sqrt{2}}\right)$ corresponding the eigenvalues $\lambda_{0}=0, \lambda_{1}=-2 p$. Since the subspace $E_{\lambda_{0}} \oplus E_{\lambda_{1}}$ has dimension $1+(p+1)=p+2$, we obtain the biharmonic index of $S^{p}\left(\frac{1}{\sqrt{2}}\right) \times S^{q}\left(\frac{1}{\sqrt{2}}\right)$ for the case $1 \leq p<q=m-p$ and $q>2 p$, which 
complete the proof of Statement (i).

For Statement (ii), first note that the proper biharmonic hypersurface $S^{m}\left(\frac{1}{\sqrt{2}}\right) \rightarrow$ $S^{m+1}$ is totally umbilical with $|A|^{2}=m, H=-1$, and hence $A(\nabla f)=-\nabla f$. It follows that the index form (17) in this case reads

$$
\begin{aligned}
Q(f) & =\int_{S^{m}\left(\frac{1}{\sqrt{2}}\right)}\left\{(\Delta f)^{2}+4|\nabla f|^{2}-4 m^{2} f^{2}\right\} \mathrm{d} v_{g} \\
& =\int_{S^{m}\left(\frac{1}{\sqrt{2}}\right)}\left\{(\Delta f)^{2}-4 f \Delta f-4 m^{2} f^{2}\right\} \mathrm{d} v_{g},
\end{aligned}
$$

where the second equality was obtained by using the divergence theorem.

Using the eigenvalues of the Laplacian on $S^{m}\left(\frac{1}{\sqrt{2}}\right)$ :

$$
\cdots<\cdots<\lambda_{2}<\lambda_{1}=-2 m<\lambda_{0}=0 \text {. }
$$

one can check that for any eigenfunction function $f$ of the eigenvalue $\lambda_{1}=-2 m$, we have $\Delta f=-2 m f$, and hence (21) becomes

$$
Q(f)=\int_{S^{m}\left(\frac{1}{\sqrt{2}}\right)}\left[(\Delta f)^{2}-4 f \Delta f-4 m^{2} f^{2}\right] \mathrm{d} v_{g}=8 m \int_{S^{m}\left(\frac{1}{\sqrt{2}}\right)} f^{2} \mathrm{~d} v_{g}>0
$$

Similarly, one can check that $Q(f)$ is positive on any other eigenspace, so the only subspace of $C^{\infty}\left(S^{m}\left(\frac{1}{\sqrt{2}}\right)\right)$ on which $Q(f)<0$ is $\mathbb{R}$ which has dimension one. Thus, we have Ind $\left(S^{m}\left(\frac{1}{\sqrt{2}}\right)\right)=1$.

Remark 2. Note that Statement (ii) in Theorem 3.1 was proved in [9] in a quite different way.

We end the paper with the following table which gives the indices of biharmonic hypersurfaces of spheres in small dimensions. 


\begin{tabular}{|c|c|c|}
\hline Ambient sphere & Biharmonic hypersurface & Index \\
\hline$S^{4}$ & $S^{1}\left(\frac{1}{\sqrt{2}}\right) \times S^{2}\left(\frac{1}{\sqrt{2}}\right)$ & 1 \\
\hline$S^{5}$ & $S^{1}\left(\frac{1}{\sqrt{2}}\right) \times S^{3}\left(\frac{1}{\sqrt{2}}\right)$ & 3 \\
\hline$S^{6}$ & $S^{1}\left(\frac{1}{\sqrt{2}}\right) \times S^{4}\left(\frac{1}{\sqrt{2}}\right)$ & 3 \\
& $S^{2}\left(\frac{1}{\sqrt{2}}\right) \times S^{3}\left(\frac{1}{\sqrt{2}}\right)$ & 1 \\
\hline$S^{7}$ & $S^{1}\left(\frac{1}{\sqrt{2}}\right) \times S^{5}\left(\frac{1}{\sqrt{2}}\right)$ & 3 \\
& $S^{2}\left(\frac{1}{\sqrt{2}}\right) \times S^{4}\left(\frac{1}{\sqrt{2}}\right)$ & 1 \\
\hline$S^{8}$ & $S^{1}\left(\frac{1}{\sqrt{2}}\right) \times S^{6}\left(\frac{1}{\sqrt{2}}\right)$ & 3 \\
& $S^{2}\left(\frac{1}{\sqrt{2}}\right) \times S^{5}\left(\frac{1}{\sqrt{2}}\right)$ & 4 \\
& $S^{3}\left(\frac{1}{\sqrt{2}}\right) \times S^{4}\left(\frac{1}{\sqrt{2}}\right)$ & 1 \\
\hline$S^{9}$ & $S^{1}\left(\frac{1}{\sqrt{2}}\right) \times S^{7}\left(\frac{1}{\sqrt{2}}\right)$ & 3 \\
& $S^{2}\left(\frac{1}{\sqrt{2}}\right) \times S^{6}\left(\frac{1}{\sqrt{2}}\right)$ & 4 \\
& $S^{3}\left(\frac{1}{\sqrt{2}}\right) \times S^{5}\left(\frac{1}{\sqrt{2}}\right)$ & 1 \\
\hline$S^{10}$ & $S^{1}\left(\frac{1}{\sqrt{2}}\right) \times S^{8}\left(\frac{1}{\sqrt{2}}\right)$ & 3 \\
& $S^{2}\left(\frac{1}{\sqrt{2}}\right) \times S^{7}\left(\frac{1}{\sqrt{2}}\right)$ & 4 \\
& $S^{3}\left(\frac{1}{\sqrt{2}}\right) \times S^{6}\left(\frac{1}{\sqrt{2}}\right)$ & 1 \\
& $S^{4}\left(\frac{1}{\sqrt{2}}\right) \times S^{5}\left(\frac{1}{\sqrt{2}}\right)$ & 1 \\
\hline
\end{tabular}

It follows from the table and Theorem 3.1 that for any natural number $k$ except $k=2$, there exists a proper biharmonic hypersurface in a sphere $S^{m}$ with $m$ depending on $k$ whose index is $k$.

\section{REFERENCES}

[1] L. Alias, On the stability index of minimal and constant mean curvature hypersurfaces in spheres, Rev. Un. Mat. Argentina, 47(2) (2006), 39-61.

[2] L. J. Alías, A. Brasil Jr. and O. Perdomo, On the stability index of hypersurfaces with constant mean curvature in spheres, Proc. Amer. Math. Soc., 135(11)(2007), 3685-3693.

[3] A. Balmus, S. Montaldo, C. Oniciuc, Classification results for biharmonic submanifolds in spheres, Israel J. Math. 168 (2008), 201-220.

[4] R. Caddeo, S. Montaldo, and C. Oniciuc, Biharmonic submanifolds of $S^{3}$, Internat. J. Math. 12 (2001), no. 8, 867-876.

[5] R. Caddeo, S. Montaldo and C. Oniciuc, Biharmonic submanifolds in spheres, Israel J. Math. 130 (2002), 109-123.

[6] B. Y. Chen, Some open problems and conjectures on submanifolds of finite type, Soochow J. Math. 17 (1991), no. 2, 169-188.

[7] G. Y. Jiang, 2-Harmonic maps and their first and second variational formulas, Chin. Ann. Math. Ser. A, 7(1986), 389-402. 
[8] G. Y. Jiang, Some non-existence theorems of 2-harmonic isometric immersions into Euclidean spaces, Chin. Ann. Math. Ser. A, 8 (1987) 376-383.

[9] E. Loubeau and C. Oniciuc, The index of biharmonic maps in spheres, Compositio Math.141 (2005),729-745.

[10] L. Mazet and H. Rosenberg, Minimal hypersurfaces of least area, J. Diff. Geom., 106(2) (2017), 283-316.

[11] Y. -L. Ou, On conformal biharmonic immersions, Ann. Global Anal. Geom. 36, 2009, 133-142.

[12] Y. -L. Ou, Biharmonic hypersurfaces in Riemannian manifolds, Pacific J. of Math, 248(1), 2010, 217-232.

[13] Y. -L. Ou and B. -Y. Chen, Biharmonic submanifolds and biharmonic maps in Riemasnnian geometry, World Scientific, Hackensack, N. J., 2020.

[14] J. Simons, Minimal Varieties in Riemannian manifolds, Ann. of Math. 88 (1968), pp 62-105.

[15] A. E. Soufi, Applications harmoniques, immersions minimales et transformations conformes de la sphère, Compositio Math. 85 (1993), 281-298.

[16] F. Urbano, Minimal surfaces with low index in the three-dimensional sphere, Proc. Amer. Math. Soc. 108 (1990), 989-992.

Department of MATHEMATics,

Texas A \& M University-Commerce,

Commerce, TX 75429,

USA.

E-MAIL:YELIN.OU@TAMUC.EDU 